\title{
Mild fetal hydronephrosis indicating vesicoureteric reflux
}

\author{
G Marra, G Barbieri, C Moioli, B M Assael, G Grumieri, M L Caccamo
}

\begin{abstract}
The management of neonates with mild hydronephrosis diagnosed antenatally is still debated. Although some of these infants are normal, it is recognised that others will have mild obstruction of the ureteropelvic junction or vesicoureteric reflux (VUR). A prospective study was performed in all newborn infants with an antenatal diagnosis of mild hydronephrosis (47 babies, 62 kidneys) born over a two year period in order to assess the frequency of VUR. Voiding cystography in 14 patients with 21 renal units showed VUR. Two patients underwent surgery and the VUR resolved; the other 12 received medical treatment. Repeat cystography was scheduled for 12-18 months later, when a high rate of spontaneous cure was observed. The remaining patients were monitored by ultrasonography but only in one case did hydronephrosis deteriorate because of the presence of severe ureteropelvic junction obstruction. It is concluded that mild dilatation of the pelvis might be an expression of a potentially severe malformation such as VUR, and a careful follow up of these cases is mandatory. (Arch Dis Child 1994; 70: F147-F150)
\end{abstract}

Mild hydronephrosis (pelvic diameter 0.5-1 $\mathrm{cm}$ ) is frequently observed at prenatal ultrasonography and does not pose immediate problems of diagnostic procedures or surgical correction at birth. Its pathological significance is uncertain, and it has been considered by some authors as a normal physiological variation or the result of transient fetal obstruction of the ureteropelvic junction. ${ }^{1}$ However, it could also be due to mild obstruction at this site or to vesicoureteric reflux (VUR) ${ }^{23}$ In the former case the obstruction can be monitored

Paediatrics and

Neonatology,

University of Milan

Medical School

G Marra

G Barbieri

C Moioli

B M Assael

M L Caccamo

Division of Paediatric

Radiology, Istituti

Clinici di

Perfezionamento,

Milan, Italy

G Grumieri

Correspondence to:

Dr Giuseppina Marra,

Clinica Pediatrica II, Via

Commenda 9, Milano, Italy.

Accepted 4 August 1993

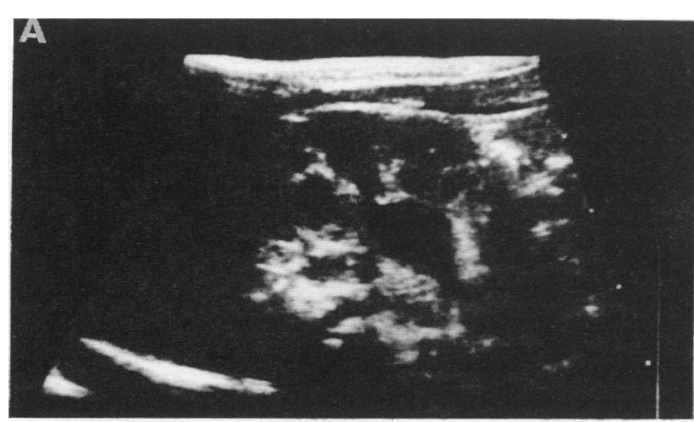
section. by postnatal ultrasonography, whereas VUR can only be diagnosed by voiding cystography. The latter investigation has never been performed systematically in this patient population so that the real pathogenicity of mild hydronephrosis is still unknown.

One of the aims of prenatal diagnosis is to detect potentially pathological situations so as to prevent or limit future damage by appropriate therapeutic measures. In the case of VUR urinary tract infections, which are the main causes of renal damage, could be prevented.

To evaluate the frequency of VUR in patients with mild hydronephrosis and whether screening for VUR is justified, we performed cystography on all infants with a prenatal diagnosis of mild hydronephrosis born at our hospital over a 24 month period. Oral informed consent was obtained from the parents.

\section{Patients and methods}

Between June 1989 and June 1991 voiding cystography was performed in all infants born in our department with mild hydronephrosis diagnosed in utero and confirmed in the first week of life. Mild hydronephrosis was defined as anteroposterior pelvic diameter in transverse section of $0.5-1 \mathrm{~cm}$ (figure). ${ }^{2} \mathrm{We}$ excluded babies who also had other urological problems such as ureter or. bladder dilatation, renal dysplasia, and more complex urological malformations at the contralateral side, as cystography is already indicated in these cases.

A total of 47 patients with 62 renal units were admitted to the study. They were all born at term (gestational age $>37$ weeks) with appropriate weight for gestational age. None of them presented with clinically important problems in the perinatal period and none of the parents and siblings were known to have VUR. Cystography was performed

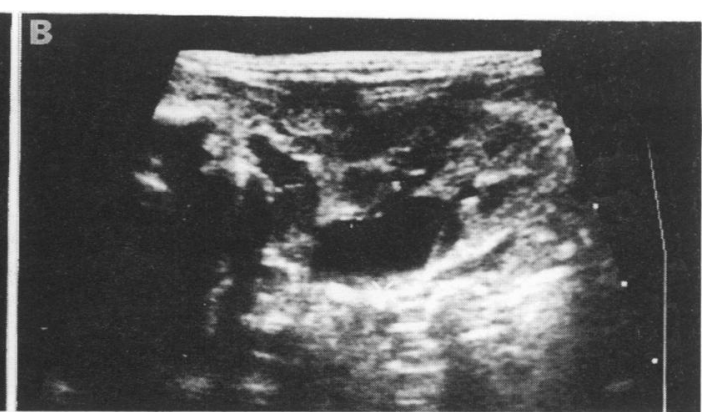

Sonograms showing mild dilatation of the pelvis. Anteroposterior pelvic diameter of $0.5 \mathrm{~cm}(A)$ and $1 \mathrm{~cm}(B)$ in transverse 
immediately after postnatal ultrasound confirmation of the dilatation, between the first week and first month of life. Antibiotic prophylaxis with trimethoprim in a single night time dose $(0.5 \mathrm{mg} / \mathrm{kg})$ was given to all the children with proved VUR. Repeat cystography to ascertain whether VUR was still present was scheduled at 12-18 months of treatment. During this period monthly urine cultures were performed in all cases routinely as well as in the presence of fever so that any urinary tract infection could be treated promptly. Blood tests to evaluate renal function and antibiotic toxicity were done at about nine month intervals.

Patients without VUR but with persistent mild dilatation underwent ultrasonography every 6-12 months (mean follow up: 20 months) and if conditions deteriorated scintigraphy or intravenous urography was planned.

\section{Results}

The 47 patients with mild hydronephrosis were enrolled in our study and included 43 boys ( 56 renal units) and four girls (six renal units). Cystography revealed the presence of VUR in 14 patients $(29.7 \%), 13$ boys (20 refluxing renal units, RRU) and one girl (one RRU). VUR was graded between $I$ and V according to the International Reflux Committee Study (grade I in one, II in three, III in eight, IV in eight, $\mathrm{V}$ in one).

Only in 14 RRU was the side of hydronephrosis the same as that of the VUR. In the other seven a VUR was present in the non-dilated contralateral renal unit. In four of them unilateral dilatation was associated with bilateral VUR, but in the other three VUR did not involve the hydronephrotic kidney but the contralateral one.

Most of the VUR was grade III or lower (one grade IV, two grade III, three grade II, one grade I). After the diagnosis of VUR 12 patients (18 RRU) received medical treatment and two (three RRU) underwent surgical correction within the second month of life. This difference in treatment was due not to selection but to referral pattern. The first follow up cystography after diagnosis has now been performed in all patients.

The long term results are reported in the table. VUR resolved in the two patients (three RRU) who underwent surgery. A spontaneous resolution was observed during the 18 months of follow up in nine of the $18 \mathrm{RRU}$, and antibiotic prophylaxis was stopped in these subjects.

During the follow up period (mean 24 months) there were two episodes of acute

Resolution during 18 months of follow up in $21 R R U$ diagnosed neonatally

\begin{tabular}{lllllll}
\hline & & \multicolumn{5}{c}{ Grade of resolved VUR/total VUR } \\
\cline { 3 - 7 } Treatment & Cure rate & $I$ & $I I$ & $I I I$ & $I V$ & $V$ \\
\hline Surgical & $3 / 3$ & - & - & $1 / 1$ & $2 / 2$ & - \\
Medical & $9 / 18$ & $1 / 1$ & $3 / 3$ & $4 / 7^{\star}$ & $1 / 6 \dagger$ & $-11 \neq$
\end{tabular}

*Final grade: I in two and III in one renal unit.

tFinal grade: II in one and IV in the others.

$¥$ Final grade: $V$. pyelonephritis for a mean frequency of 0.006 infections/year/patient. None of the children presented with acidosis, polyuria, increased serum creatinine, or altered urinary values. The dilatation worsened only in one patient of the 33 in whom mild hydronephrosis was not associated with VUR. This patient was operated on after 12 months for an ureteropelvic obstruction that had been seen on intravenous urography.

\section{Discussion}

The clinical importance of mild hydronephrosis is uncertain ${ }^{12}$ and the detail and timing of investigations and follow up are still controversial. $^{24}$ Cases have been reported of VUR diagnosed after urinary tract infections in patients with mild fetal hydronephrosis who were not followed up. ${ }^{5}$ To our knowledge the present study is the first to investigate the frequency of reflux in a large series and it indicates that in patients with slight renal pelvic dilatation diagnosed in fetal life the probability of finding VUR is about $30 \%$. The frequency of VUR observed by us is certainly raised and much higher than that estimated in the general paediatric population, which is between $0.4 \%$ and $1.8 \%{ }^{6}$ Our finding that VUR was prevalent in boys confirms the observations of other authors who studied VUR in patients with a prenatal diagnosis of hydronephrosis. ${ }^{2}{ }^{7-9}$ In a pathological study of 747 necropsies Cussen reported a threefold greater prevalence of VUR in boys than girls, ${ }^{10}$ and it was tested by ligating the urethra and infusing saline into the bladder. This contrasts with the results of studies performed before the advent of prenatal diagnosis in which the prevalence of VUR appeared greater in girls. ${ }^{11}$ The discrepancy is probably related to case selection. The event leading to diagnosis of VUR in the majority of the cases was urinary tract infection, which is more frequent in girls. ${ }^{12}$ In family studies on VUR the sex ratio has been equal; in these studies the selection of cases is not biased by mode of presentation. ${ }^{13}$

As already observed by Steele et $a l,{ }^{7}$ the course of VUR in our patients given medical treatment was benign. The frequency of urinary tract infections was low and a high proportion of cases underwent spontaneous resolution within the first 18 months (table).

The outcome in the absence of diagnosis and treatment is a matter of conjecture. Screening for a disease is justified when the population screened is at real risk for the disease and early diagnosis can prevent its most serious consequences. VUR could fulfil these conditions in patients with hydronephrosis diagnosed in fetal life, as potential damage could be limited by prevention of urinary tract infections. ${ }^{14}$ According to our findings, the need for cystographic screening for all babies with an antenatal diagnosis of mild hydronephrosis must be evaluated as this procedure is invasive and expensive and exposes the child to radiation. In our opinion, careful ultrasound monitoring during the first month of life is important for all infants in 
whom hydronephrosis has been diagnosed before birth. If the presence of hydronephrosis is confirmed, various approaches can be considered. Based on our data, the routine use of cystography would be useless in $70 \%$ of the cases. As all our patients were treated with antibiotic prophylaxis once VUR had been diagnosed, we do not know how many episodes of infections or how many scars were prevented by the early recognition of the malformation, so we cannot comment on the cost benefit ratio of early cystography. Alternatively the patient with a mild dilatation can be followed up without cystography. In this case urinary tract infections should be monitored the family informed of the risks and symptoms of these infections and of VUR. However, cystography seems indicated in cases with additional risk factors such as poor compliance with follow up and where distance restricts access to health care.

This study was supported financially by Associazione del Bambino Nefropatico, Milan, Italy and by Regione Lombardia.

1 Homsy YL, Williot P, Danais S. Transitional neonata hydronephrosis: fact or fantasy? $\mathcal{F}$ Urol 1986; 136: 339-41.

2 Thomas DFM, Gordon AL. Management of prenatally diagnosed uropathies. Arch Dis Child 1989; 64: 58-63.

Fine R. Diagnosis and treatment of fetal urinary tract abnormalities. F Pediatr 1992; 121: 333-41.

4 Watson AR, Readett D, Nelson CS, Kapila L, Mayell MJ. Dilemmas associated with antenatally detected urinary tract abnormalities. Arch Dis Child 1988; 63: 719-22.

5 Smith D, Eggington JA, Brookfield DSK. Detection of abnormality of fetal urinary tract as a predictor of rena tract disease. $B M \mp 1987 ; 294: 27-8$.

6 Bailey RR. Vesicoureteric reflux in healthy infants and children. In: Hodson J, Kincaid Smith P, eds. Reflux nephropathy. New York: Masson, 1979: 57-61.

7 Steele BT, Robitaille P, De Maria J, Grignon A. Follow up evaluation of prenatally recognized vesicoureteric reflux. $\mathcal{F}$ Pediatr 1989; 115: 95-6.

8 Yeung CK, Dhillon HK, Duffy PG, Ransley PG Vesicoureteric reflux in infants with prenatally diagnosed Vesicoureteric reflux in infants with
hydronephrosis. $\mathcal{F}$ Urol $1991 ; 4: 64$.

9 Steele BT, De Maria J. A new perspective on the natura history of vesicoureteral reflux. Pediatrics 1992; 90: 30

10 Cussen LJ. Vesicoureteral reflux in children. Frequency and associated urologic abnormalities. Investigative Urology 1971; 8: 640-4.

11 Baker R, Maxted DW, Mayalath J. Relation of age, sex and infection to reflux: data indicating high spontaneous cure rate in pediatric patients. $\mathcal{F}$ Urol 1966; 95: 27.

12 Lenaghan D, Whitaker JG, Jensen F, Stephens ED. The natural history of reflux and longterm effects of reflux on the kidney. F Urol 1976; 115: 728-30.

13 Kenda RB, Fettich JJ. Vesicoureteric reflux and renal scars in asymptomatic siblings of children with reflux. Arch Dis in asymptomatic siblings

14 Smellie JM, Ransley PG, Normand ICS, Prescod N, Edwards D. Development of new renal scars; a collaborative study. BMF 1985; 290: 1957-60.

\section{Commentary}

During the past decade since the advent of routine antenatal ultrasound scanning fetal hydronephrosis has emerged as a common and potentially pathological condition. It has been suggested that a pelvicaliceal diameter of more than $5 \mathrm{~mm}$ is abnormal. In many cases fetal hydronephrosis is transient and resolves spontaneously in the latter part of pregnancy or after delivery, but in other cases the hydronephrosis persists or worsens. Mild fetal hydronephrosis may be due to mild or transient obstruction or may reflect urinary tract dilatation or vesicoureteric reflux (VUR). When the postnatal scan is done very soon after delivery the infant urine production rate is very slow and the size of the renal pelvis may be underestimated. Using the international
VUR grading system grades I and II are not associated with dilatation on cystography but grades III, IV, and V are associated with mild to severe dilatation at cystography but not necessarily on ultrasound. The screening method used in the paper by Marra et al is therefore more likely to have underestimated than overstated the prevalence of VUR.

VUR is essentially a silent condition and was initially detected only after the investigation of severe recurrent urinary tract infection often in children with grossly abnormal urinary tracts. In 1960 Hodson and Edwards demonstrated that VUR was a common finding in children with urinary tract infection particularly when kidney scars were present, ${ }^{1}$ and after their work many children with a history of urinary tract infection underwent micturating cystourethrography. As more girls than boys develop infections, particularly recurrent infections, VUR was demonstrated more often in girls, although the incidence of VUR in children with urinary tract infection has not been shown to be different between the two sexes.

Recently the observation that VUR had a familial distribution has led to family studies and the routine counselling of families about the risk of VUR and urinary tract infection in newborn infants. As Marra et al have suggested the mode of presentation or case selection may have a profound effect on the perceived incidence of VUR. When infants are investigated soon after birth the prevalence of VUR in the first degree relatives approaches $50 \% .^{23}$ The natural history of VUR is for improvement with increasing age, however, ${ }^{4}$ and it is not surprising that in those studies which included older children or adults a lower incidence of VUR was found. ${ }^{5}$

In 1971 Cussen, studying necropsies, showed that there were more cases in boys. ${ }^{6}$ However in his series the cause of death is not clearly stated. Infant boys are particularly prone to urinary tract infections and there is an increased risk of serious illness at this age. In addition, renal failure due to reflux nephropathy is more common in boys than girls in early childhood. It is therefore possible that the increased incidence of VUR in boys in his study was due to a bias in case selection. Similarly the increased incidence of VUR in boys in the paper by Marra et al may be due to the fact that mild fetal hydronephrosis is more common in infant boys. Kenda et al also observed VUR to be more common in infant boys than girls in his families. ${ }^{2}$

The incidence in girls in the community can be estimated from studies in girls with asymptomatic bacteriuria, which has a prevalence of $1-2 \%$. As asymptomatic bacteriuria is an intermittent condition it is likely that the population at risk is greater than this. A third of these girls have demonstrated VUR giving a minimum incidence of $0.5 \%$ of the female population and as VUR improves with age the incidence at birth may be much more. In boys there are no comparable data because asymptomatic infection is rare, however family studies in infants and women who had symptomatic infection in childhood have shown that infant boys are affected as often as infant girls. ${ }^{3}$ 